\title{
Automatic Extraction of Buildings in an Urban Region
}

\author{
Fasahat Ullah Siddiqui \\ Faculty of Information Technology \\ Monash University, Australia \\ fasahat.siddiqui@monash.edu
}

\author{
Shyh Wei Teng, Guojun Lu, Mohammad Awrangjeb \\ Faculty of Science and Technology \\ Federation University, Australia \\ \{shyh.wei.teng,guojun.lu, mohammad.awrangjeb\} \\ @federation.edu.au
}

\begin{abstract}
There are currently several automatic building extraction methods introduced in the literature, but none of them are capable to completely extract portions of a building that are below a predefined building minimum height threshold. This paper proposes a systematic method which analyzes the height differences between the extracted adjacent planes above and below the height threshold as well as the planes' connectivity, thereby, extracting all portions belonging to buildings more completely. In general, the height difference between the edges of the adjacent planes above and below the height threshold that belong to the same building is more uniform. In addition, the extracted planes below the height threshold that belong to a building and their adjacent ground planes also have a clear height difference. The proposed method incorporates such information to achieve better performance in building extraction. We have compared our proposed method to a current state-of-the-art building extraction method qualitatively and quantitatively. Our experimental results show that our proposed method successfully recovers portions of a building below the height threshold, thereby achieving relatively higher average completeness (an improvement of $1.14 \%$ ) and quality (an improvement of $0.93 \%$ ).
\end{abstract}

\section{Categories and Subject Descriptors}

I.4.6 [Image Processing and Computer Vision]: Segmentation region growing and partitioning. I.4.8 [Image Processing and Computer Vision]: Scene Analysis - object recognition.

\section{General Terms}

Algorithms.

\section{Keywords}

Building planes, ground planes, height difference between planes.

\section{INTRODUCTION}

Automatic building extraction is an active research topic in field of remote sensing. One of the limitations of the existing building extraction methods is the correct extraction of portions of a building whose height is similar to other low lying objects like trees, bushes and vehicles [1]. Besides not having a distinct height

\footnotetext{
Permission to make digital or hard copies of all or part of this work for personal or classroom use is granted without fee provided that copies are not made or distributed for profit or commercial advantage and that copies bear this notice and the full citation on the first page. Copyrights for components of this work owned by others than ACM must be honored. Abstracting with credit is permitted. To copy otherwise, or republish, to post on servers or to redistribute to lists, requires prior specific permission and/or a fee. Request permission from Permission@acm.org.

IVCNZ '14, November 19 - 21 2014, Hamilton, New Zealand

Copyright 2014 ACM 978-1-4503-3184-5/14/11 ..\$15.00

http://dx.doi.org/10.1145/2683405.2683449
}

difference, such portions of a building and the rest of the building might have different color intensities. This problem becomes even more complex if the building lies on a hilly area where the ground heights surrounding the building could be distinctly different $[2$, 3]. Furthermore, LIDAR data have irregular data spacing (a.k.a. irregular pattern) [4], which corrupts the 3D geometry of building and causes incomplete building extraction.

An ongoing trend for extracting building is to use LIDAR data, where co-planarity of the LIDAR points is mainly considered [5, $6]$. In fact, this approach of building extraction is to first extract 3D roof planes using co-planarity, and then extract their respective $2 \mathrm{D}$ space (area) of a building. There are two different ways to extract the 3D planes: (1) using only non-ground LIDAR points and (2) using all LIDAR points. In former way, a rulebased method is used to separate LIDAR points into ground and non-ground points $[6,7]$. Next, the co-planarity of non-ground points are tested using the Delaunay triangle neighborhood or Voronoi diagram neighborhood algorithm. After that, the planar segments are extracted and refined. In the final step, the Canny edge detector is applied to find the building's boundary and area. [6] is a method which belongs to the first way of building extraction methods described above and its authors employed a region growing method. It extracts planes based on the coplanarity of non-ground points, where co-planarity is measured using the Delaunay triangle neighborhood algorithm [5, 7]. Later, the Canny edge detector is applied on extracted planes to remove trees by analyzing plane boundaries using a set of defined rules. As this method requires manual definition of rules like height threshold and plane boundary irregularity threshold, it is highly subjective. In addition, the building portions, which have height, lower than the pre-defined threshold (e.g. 1 meter) or are small in size, are not extracted. In [7], another region growing method is used on non-ground LIDAR co-planar points, where the seed points are selected at different heights from the maximum height of the non-ground LIDAR points to pre-defined minimum height threshold. The extracted planes at different heights are merged if they are completely or partially overlapped with each other. Later, a refinement process is employed to differentiate trees and buildings. Finally, the edges of the planes that define the building areas are extracted. However, this method is also constrained by the pre-defined minimum height threshold.

The second way of extracting building planes clusters the entire co-planar LIDAR points by measuring their co-planarity using Voronoi diagram neighborhood algorithm [5]. These co-planar LIDAR points are grouped into a number of clusters with respect to the normal vector of each co-planar LIDAR point. A limitation of this method is the accuracy of the number of clusters obtained is highly sensitive to the parameters set for the pre-defined criteria. Furthermore, Fuzzy C-means clustering does not guarantee optimal convergence [8] and its convergence time might be relatively long [7]. This paper proposes a new method 
which can more accurately extract the building portions at all heights and without being restricted by a pre-defined minimum height threshold for buildings. This proposed method first extracts the ground and non-ground planes (expected building planes). Then, it analyzes these planes using pre-defined rules likes height differences among the planes and position. Finally, these planes are re-categorised into building and non-building planes. The remainder of this paper is organized as follows: Section 2 outlines the background concept of the baseline method of building extraction and its limitations. The implementation of the proposed building extraction (PBE) method is proposed in Section 3. Section 4 explains the experimental set up. Section 5 presents the qualitative and quantitative experimental results of the PBE method compared with the baseline method. Finally, Section 6 concludes the paper.

\section{BACKGROUND}

\subsection{Baseline Method}

The method in [7] is considered as the baseline method in this paper. This method uses LIDAR data and DEM (Digital elevation model) data as inputs. LIDAR data represent the height of the ground surface including all the objects on it measured from the sea-level. On the other hand, the bare earth DEM data represent the height of the bare ground surface without the objects measured from sea-level. Therefore, a manually set threshold, e.g. 1 meter, is added to the DEM data for differentiating the LIDAR data which belong to ground and those which belong to nonground. Later, the co-planarity of the non-ground LIDAR points is measured using the Delaunay triangle neighborhood algorithm. After that, lines are detected from LIDAR points at different heights and their mid-points are selected as seed points of the region growing method. Regions are grown on the non-ground coplanar LIDAR points and planes are extracted at different height levels. The overlapping planes are merged into a single plane representing a non-ground object (i.e. building or trees). Finally, the extracted planes are analyzed by using three refinement criteria (i.e. used-point ratio, object shape information, and height gap). The planes, which do not fulfill these criteria are defined as tree planes and are then removed. The boundaries of each remaining plane are extracted to define area of each building.

\subsubsection{Co-planarity of non-ground LIDAR points}

The eigenvalues of covariance matrix [5] are used to find whether a certain non-ground point is co-planar with respect to its neighborhood points or not. The eigenvalue for each non-ground LIDAR point is measured using the geometrical method known as Delaunay triangulation. Usually, the eigenvalue for co-planar points is zero. However, this is not practical due to the noise in the data. Therefore, a variation of 0.005 in eigenvalue is acceptable to consider a point as co-planar.

\subsubsection{Seed point selection}

We start the selection of the seed points at the maximum height $(\mathrm{H})$ of the LIDAR points, where LIDAR points are grouped into regions. However, LIDAR data have inherent noise and it is unlikely to get significant number of LIDAR points at certain height to generate regions. Therefore, a tolerance level $(t)$ is added into the maximum height $(\mathrm{H})$ of LIDAR points, and the height threshold (HT) is defined as:

$\mathrm{HT}=\mathrm{H} \pm \mathrm{t}$
Later, the LIDAR points with a similar height threshold (HT) are grouped into regions, and the mid-point of each region edge is detected as a seed point. Later, the maximum height of LIDAR points is updated according to Equation (2).

$\mathrm{H}=\mathrm{H}-1 / 2$ meter

Equations (1) and (2) are repeated until the value of $\mathrm{H}$ equals to the minimum height of non-ground LIDAR points.

\subsubsection{Plane extraction}

Each seed point is used as a starting point for assessing the coplanarity of it and its neighboring LIDAR non-ground points. Based on the plane equation, neighboring points which are coplanar are merged to gradually grow a plane until no new points with similar co-planarity are found. As we know, LIDAR points have no regular spatial pattern. Therefore, errors such as distance spacing error and height error are common in LIDAR points. These errors can greatly influence the merging of neighboring LIDAR points. To overcome these errors, three distinct norms are used $[6,7]$. The first norm is LIDAR point spacing which is the maximum distance between two neighboring LIDAR points. The second norm is height error which relates to random height error in neighboring non-ground LIDAR points of a plane. If the height error between the neighboring points is less than 10 centimeters, then points are considered as part of a plane. The third norm is actual distance between the non-ground LIDAR points and a plane. If the actual distance of any non-ground LIDAR points from a plane is less than 15 centimeters, then these points are considered as a part of a plane. In addition, the height variation of a plane is analyzed to remove tree planes.

\subsubsection{Refinement process to remove tree planes}

To remove the tree planes, three rule-based criteria have been used. These three criteria are as follows:

Used-point ratio: This is the ratio between the number of LIDAR points used for generating plane and the actual number of LIDAR points in a plane-occupied region. Relatively, trees planes accumulate much fewer LIDAR points for generating plane and can be easily differentiated from building plane based on the used-point ratio. The used-point ratio is set at $60 \%$, and planes are removed if their used-point ratio is less than this threshold.

Object shape information: Plane shape information like area and width are very useful for differentiating the trees planes from the building planes. Relatively, a tree consists of many small planes and they are removed if their width is less than 1 meter. In addition, isolated planes (i.e. object with single plane) are removed if their area is less 5 meter square, and non-isolated planes (i.e. object with many planes) are also removed if their area is less than 1 meter square.

Height gap: Height gap is the height difference between two neighboring planes of an object (e.g. building and tree). A tree has relatively many more planes and the height gaps between these planes are greater than the height gaps between the planes of a building. Therefore, tree planes with height gaps more than the pre-defined threshold are removed.

\subsubsection{Limitation of the baseline method}

As described above, the baseline method uses a pre-defined minimum height threshold to eliminate the LIDAR points of ground objects such as vehicles, gardens, and bushes. Using such 
a height threshold is a limitation as many LIDAR points which represent buildings might also be eliminated. The following are two cases where such incorrect elimination would happen: (1) in a building which has portions of the building lower than the predefined height threshold. Examples of such portions of a building are car parking garage and shed; (2) at hilly areas where buildings are lying on highly uneven ground. Incorrect ground height information is common for an uneven ground which causes relatively low height estimation of hilly area buildings. By employing a pre-defined height threshold, the LIDAR points of those buildings are easily eliminated.

We illustrate Case (1) with an example shown in Figure 1. There are two buildings in the area bounded by a blue box. The building on the right is a multi-height building and some portions of this building are below 1 meter. Therefore, if the pre-defined height threshold is set at 1 meter, such portions of the building will not be extracted as parts of the building by the baseline method. An example of a portion of the building that the baseline method failed to correctly extract is marked by a yellow arrow.

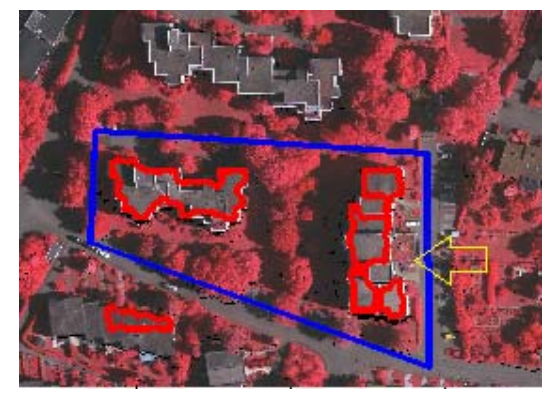

Figure 1. Missing of low height building after applying the baseline method.

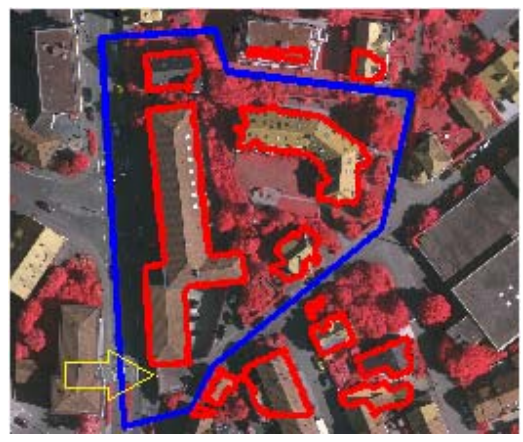

Figure 2. Missing of low height building on hilly area after applying the baseline method.

Case (2) is illustrated with an example as shown in Figure 2. There are three buildings in the area bounded by a blue box. The building on the bottom-left is lying on a hilly area (i.e. extremely uneven ground) and a portion of it has incorrect height (i.e. below 1 meter) with respect to the uneven ground. Therefore, if the predefined height threshold is set at 1 meter, such potions of the building will not be extracted by the baseline method. An example of a portion of the building that the baseline method failed to correctly extract is marked by a yellow arrow in Figure 2.

\section{PROPOSED METHOD}

As stated in the previous sections, it is a standard practice in building extraction to manually set a pre-defined minimum height threshold (e.g. 1 meter) and consider the LIDAR points equal or above this threshold to be potential candidates for representing buildings. Due to this, LIDAR points lower than the height threshold are filtered out by a building extraction method and are unlikely to be analyzed again to determine if they represent a building. We have already illustrated this limitation with examples in Figures 1 and 2.

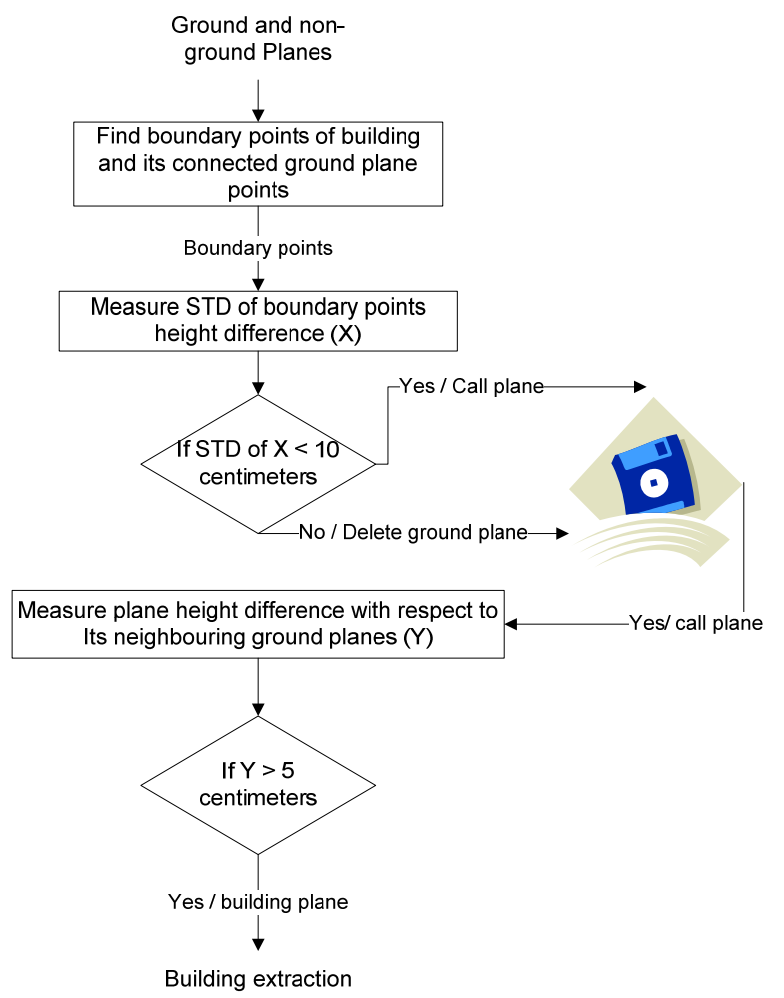

Figure 3. An Implementation of analyzing steps of the proposed method.

In our proposed building extraction (i.e. PBE), the height difference and connectivity between the extracted building planes and their neighboring ground planes are analyzed. Analyzing the aforementioned characteristics ensures the better extraction of low height portions of the buildings. The main steps of the proposed method are as follows:

1) Extract building planes from non-ground points using the baseline method.

2) Extract ground planes from ground co-planar points using region growing method (the same method used in the baseline method for plane extraction).

3) Extract building-connected-ground planes using the below steps:

a) Find the boundary points of buildings planes and their connected ground planes.

b) Measure height differences between boundary points of building planes and their connected ground planes. Later, the height differences are stored in variable X.

c) Calculate the standard deviation of variable $X$, and if it is less than pre-defined threshold, then ground planes which are connected to buildings are assumed as low building portions. 
4) Analyze building-connected-ground planes using the below steps:

a) Measure height difference between the buildingconnected-ground planes and their neighboring ground planes. The derived height differences are stored in variable Y.

b) If all stored values of variable $Y$ are greater than predefined threshold, then store building-connected-ground planes (i.e. low height building planes).

5) Finally, merge building-connected-ground planes into building planes.

The implementation of proposed steps for extracting buildings is depicted in Figure 3. The main steps of the proposed method (i.e. extraction of building-connection-ground plane and the analysis of the building-connected-ground planes) are further described with an example in Sections 3.1 and 3.2.

\subsection{Extraction of Building-Connected Plane}

Usually, the building portions which are below the height threshold are connected to building plane. Furthermore, the height difference between a building plane and a low building portion is the same on their connected side (i.e. common side). Based on the plane connectivity, the boundary points of the building plane and their connected ground planes are extracted. As shown in Figure 4(c), the points of left building (marked by blue) and the points of left building connected ground planes (marked by red) are extracted. Later, the height difference between the boundary points of building and its connected ground planes is measured, which is denoted by variable $X$. If the standard deviation of $X$ is less than pre-defined threshold, then the ground planes (which are highlighted by red color in Figure 4(d)) are assumed as low height building portion and stored in a library.



(a)

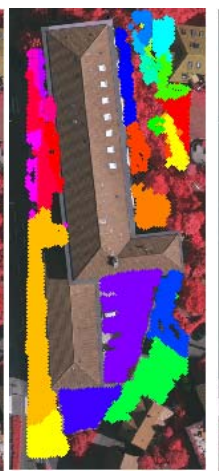

(b)



(c)

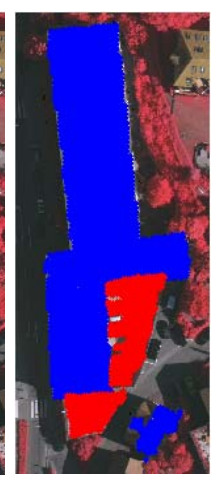

(d)
Figure 4. Extracted planes of (a) buildings and (b) their connected ground, (c) boundary points of buildings and their connected planes, and (d) buildings and their connected ground objects planes.

\subsection{Analyze Building-Connected Plane}

To verify low height building portions, the building's heights with respect to their adjacent ground planes are measured and is denoted by variable $\mathrm{Y}$. If the $\mathrm{Y}$ is more than pre-defined threshold, then the low building portion plane is confirmed as building plane. Later, the planes which are lying above certain height threshold are added into building plane and are completely extracted as the building area from site. This step is illustrated by an example in Figure 5(a), where the red portions of building are analyzed with respect to their neighboring ground planes. As shown, the red building portion at bottom-most of Figure 5(a) is now marked as building plane in blue as it is above the height threshold. The red false building plane of Figure 5(b) is removed and only the blue building planes are left which is shown in Figure 5(c).

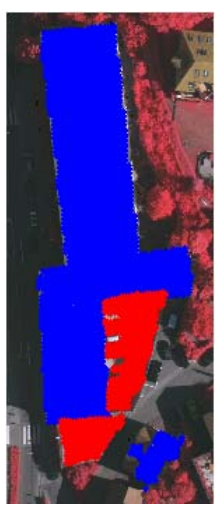

(a)

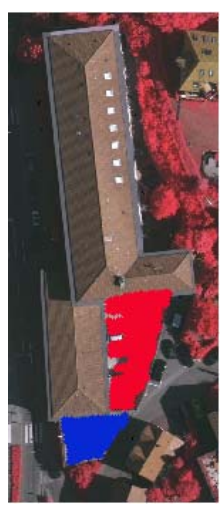

(b)

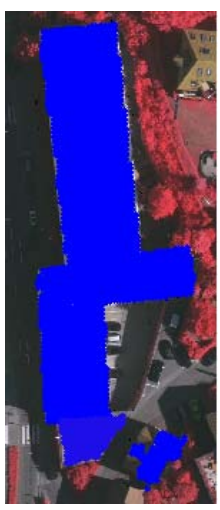

(c)
Figure 5(a) building portions which are lying on ground plane, (b) blue is low height buildings portion and red is false building portion, and (c) and building planes at all height level.

\section{EXPERIMENTAL SETUP}

The three areas (i.e. VA01, VA02, and VA03) of the Vaihingen site of Germany are used to evaluate the performance of proposed method. The Germany site has been adopted by the ISPRS benchmark [9]. The LIDAR point density of areas varies from 4 to 6.7 points per meter square.

Table 1. Parameters set for the baseline method and proposed PBE method.

\begin{tabular}{|c|c|c|c|}
\hline Process & Parameter & $\begin{array}{c}\text { baseline } \\
\text { method }\end{array}$ & PBE \\
\hline $\begin{array}{l}\text { Filter out non- } \\
\text { ground LIDAR } \\
\text { point }\end{array}$ & $\begin{array}{l}\text { Height } \\
\text { threshold }\end{array}$ & $1 \mathrm{~m}$ & $1 \mathrm{~m}$ \\
\hline Co-planarity & $\begin{array}{l}\text { Eigenvalue } \\
\text { Variation } \\
\end{array}$ & 0.005 & 0.005 \\
\hline \multirow{2}{*}{ Plane extraction } & Height error & $10 \mathrm{~cm}$ & $10 \mathrm{~cm}$ \\
\hline & Normal distance & $15 \mathrm{~cm}$ & $15 \mathrm{~cm}$ \\
\hline \multirow{5}{*}{ Refinement } & Used-point ratio & $60 \%$ & $60 \%$ \\
\hline & Object length & $1 \mathrm{~m}$ & $1 \mathrm{~m}$ \\
\hline & $\begin{array}{l}\text { Isolated object } \\
\text { area }\end{array}$ & $1 \mathrm{~m}^{2}$ & $1 \mathrm{~m}^{2}$ \\
\hline & $\begin{array}{l}\text { Non-isolated } \\
\text { object area }\end{array}$ & $5 \mathrm{~m}^{2}$ & $5 \mathrm{~m}^{2}$ \\
\hline & Height gap & $15 \mathrm{~cm}$ & $15 \mathrm{~cm}$ \\
\hline $\begin{array}{l}\text { Building connective } \\
\text { plane extraction }\end{array}$ & $\begin{array}{l}\text { Threshold for } \\
\text { standard } \\
\text { deviation } \\
\text { of variable } \mathrm{X}\end{array}$ & - & $10 \mathrm{~cm}$ \\
\hline $\begin{array}{l}\text { Low height building } \\
\text { connective plane }\end{array}$ & $\begin{array}{l}\text { Threshold for } \\
\text { variable Y }\end{array}$ & - & $5 \mathrm{~cm}$ \\
\hline
\end{tabular}



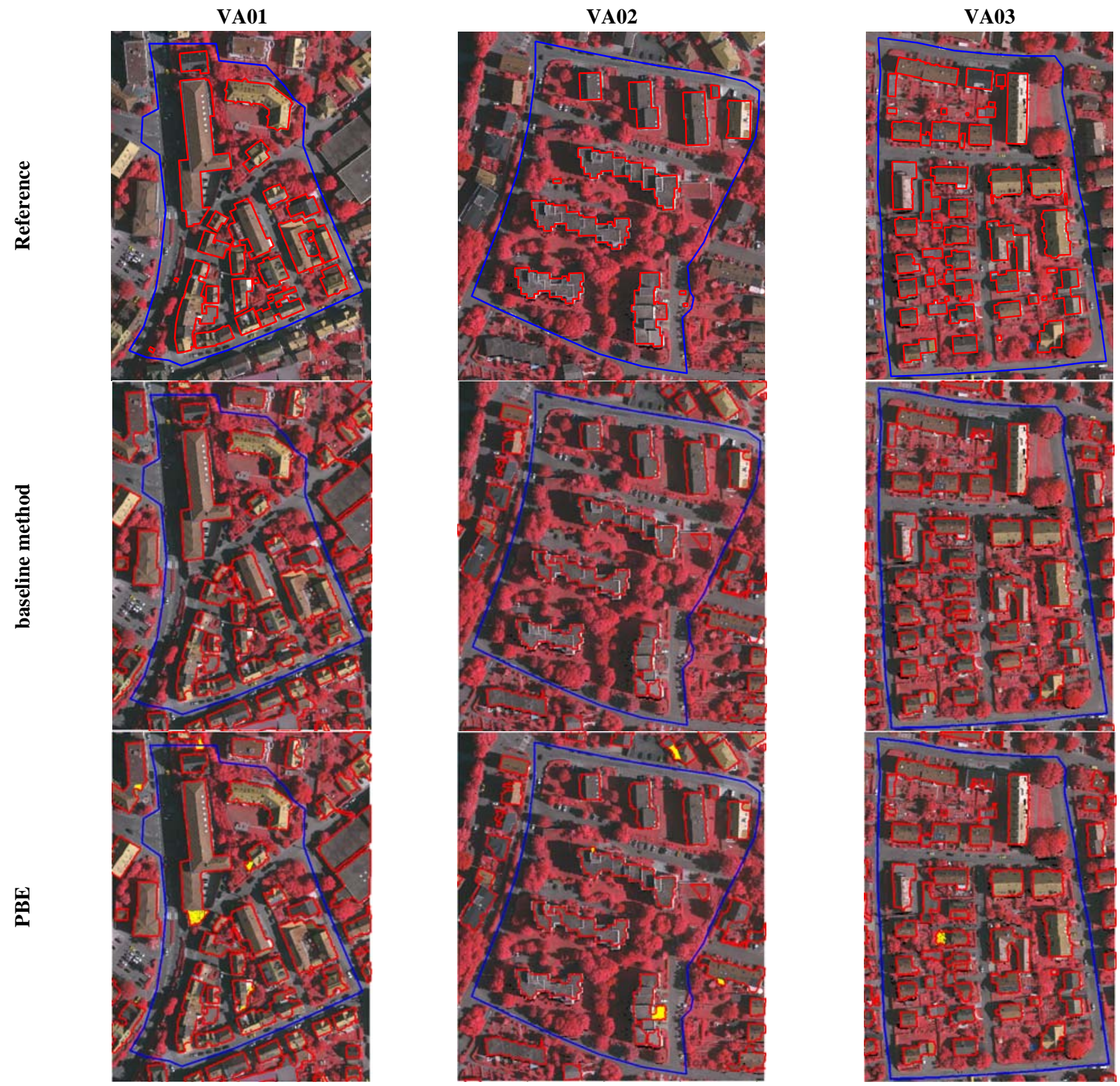

Figure 6. Results generate after applying the baseline and proposed methods on the German areas.
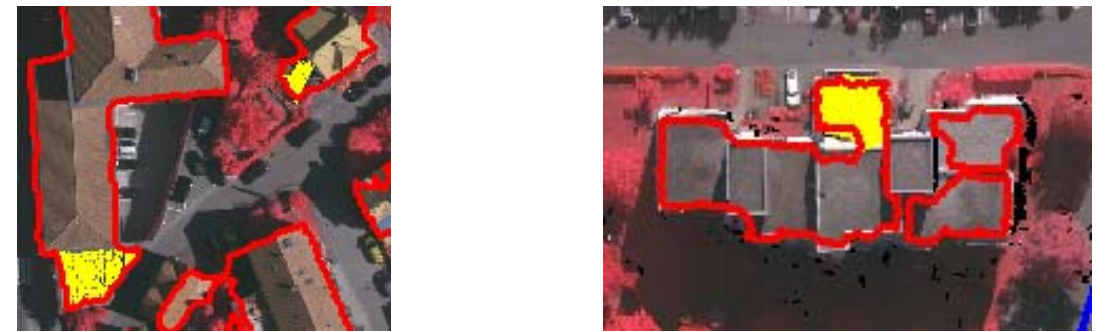

Figure 7. Magnified view of the low height building portions in (left) VA01 and (right) VA02 sites that are extracted by PBE method.

To evaluate the performance of our proposed method, the reference benchmark is obtained using the Barista software [10]. Parameters are set for the baseline and the PBE methods. The complete list of parameter settings used in the experiments presented in this paper for the baseline and the PBE methods are shown in Table 1. The pixel-based evaluation method is adopted to compare the performance of proposed method with the baseline method. For the pixel-based evaluation method, pixels in a photogrammetric image are used for deriving more accurate evaluation indices. In this evaluation method, the True Positive (TP), False Negative (FN), and False Positive (FP) indices indicate the rate of correct buildings, missing buildings, and non- 
buildings portion detected by a method respectively. On the other hand, the completeness $(\mathrm{Cm})$, correctness $(\mathrm{Cr})$, quality $(\mathrm{Q})$ indices are also used to verify the effectiveness of a method. These indices are defined as [11]:

$$
\begin{aligned}
& C m=\frac{T P}{T P+F N} \% \\
& C r=\frac{T P}{T P+F P} \% \\
& Q=\frac{T P}{T P+F P+F N} \%
\end{aligned}
$$

\section{RESULT AND DISCUSSION}

The three areas of the German site in the data-set are tested and the generated results are shown in Figure 6. Qualitative and quantitative analyses are performed to evaluate the performance of proposed building extraction method as compared to the baseline method. In the qualitative analysis, the ISPRS benchmark defined regions of interest are marked in dark blue, whereas the extracted building regions are marked in red. Evaluating qualitatively, the low building portions are successfully extracted by the PBE method as compared to the baseline method. The low building portions are highlighted in yellow as shown in Figure 6. For better visual evaluation, the key areas of PBE generated results are magnified. The lower height portions of buildings (highlighted in yellow) are completely extracted by PBE, which are also shown in Figure 7.

Table 2. Results based on Pixel-based evaluation.

\begin{tabular}{|c|l|l|l|l|c|}
\hline Indices & Method & VA01 & VA02 & VA03 & Average \\
\hline \multirow{2}{*}{ TP } & PBE & 765257 & 561231 & 946167 & 757551.7 \\
\cline { 2 - 6 } & baseline & 752190 & 550672 & 946167 & 749676.3 \\
\hline \multirow{2}{*}{ FN } & PBE & 143717 & 82321 & 210562 & 145533.3 \\
\cline { 2 - 6 } & baseline & 135971 & 79244 & 209290 & 141501.7 \\
\hline \multirow{2}{*}{ FP } & PBE & 40279 & 32097 & 59159 & 43845.00 \\
\cline { 2 - 6 } & baseline & 53346 & 42656 & 59159 & 51720.30 \\
\hline \multirow{2}{*}{ Cm\% } & PBE & 95.0 & 94.6 & 94.1 & 94.57 \\
\cline { 2 - 6 } & baseline & 93.4 & 92.8 & 94.1 & 93.43 \\
\hline \multirow{2}{*}{ Cr\% } & PBE & 84.2 & 87.2 & 81.8 & 84.40 \\
\cline { 2 - 6 } & baseline & 84.7 & 87.4 & 81.9 & 84.67 \\
\hline \multirow{2}{*}{ Q\% } & PBE & 81.6 & 83.1 & 77.8 & 80.83 \\
\cline { 2 - 6 } & baseline & 79.9 & 81.9 & 77.9 & 79.90 \\
\hline \multirow{2}{*}{ Time } & PBE & 5423.31 & 6966.37 & 4869.49 & 5753.06 \\
\cline { 2 - 6 } (sec) & baseline & 3722.56 & 5569.44 & 3317.35 & 4203.11 \\
\hline
\end{tabular}

In addition, evaluating quantitatively, the TP, FP, $\mathrm{Cr}$ and $\mathrm{Q}$ values in Table 2 also show that the proposed method is more robust in extracting buildings that have low height in VA01 and VA02 areas of German site. The TP values clearly show that a greater total area of the buildings is extracted by the PBE method. On the other hand, as the VA03 area does not have the low building portion, its TP value is similar for the PBE and the baseline methods. In addition, the completeness and quality for all areas of German site are also improved by $1.14 \%$ and $0.93 \%$ respectively using the PBE method. Finally, the computing time required by the baseline and the proposed methods are also shown in the lasts two row of Table 2 .

\section{CONCLUSION}

In this paper, additional steps are proposed to analyze the ground planes which are connected to the building planes and to extract the building portions that have low height. In addition, the proposed method is compared to a state-of-the-art method for building extraction. Our experimental results show that the defined criteria in additional steps of our proposed method are successful in extracting buildings with all their premises (i.e. low height car parking garage and shed). It is also confirmed by the quantitative results, where the completeness and quality is improved by $1.14 \%$ and $0.93 \%$ respectively.

\section{REFERENCES}

[1] Ren, Z., Zhou, G., Cen, M., Zhang, T., and Zhang, Q. 2008. A novel method for extracting building from lidar data Fc-S method. The International Archives of the Photogrammetry, Remote Sensing and Spatial Information Sciences (Beijing 2008).

[2] Awrangjeb, M., Ravanbakhsh, M., and Fraser, C. S. 2010. Automatic detection of residential buildings using LIDAR data and multispectral imagery. ISPRS J. Photogramm. 65, 5(2010) 457-467.

[3] Parvathi, K., Prakasa Rao, B. S., Venkateswara Rao, T., and Mruthyunjaya Reddy, K. 2010. Feature extraction from satellite images of hilly terrains using wavelets and watersheds, ISPRS J Photogramm. 31(2010) 5855-5866. DOI=http://dx.doi.org/10.1080/01431161.2010.512309.

[4] Li, Y., and $\mathrm{Wu}, \mathrm{H}$. 2008. Adaptive building edge detection by combining LiDAR data and aerial images. The nternational Archives of the Photogrammetry, Remote Sensing and Spatial Information Sciences (Beijing 2008).

[5] Sampath, A., and Shan, J. 2010. Segmentation and reconstruction of polyhedral building roofs from aerial LIDAR point clouds. IEEE Geosci. Remote. S. 48, 3(Mar. 2010) 1554-1567.

DOI= http://dx.doi.org/10.1109/TGRS.2009.2030180.

[6] Awrangjeb, M., Zhang, C., and Fraser, C. S. 2013. Automatic extraction of building roofs using LIDAR data and multispectral imagery. ISPRS J. Photogramm. 83 (2013)1-1883.

DOI $=$ http://dx.doi.org/10.1016/j.isprsjprs.2013.05.006.

[7] Abdullah, S., Awrangjeb, M., and Lu, G. Automatic segmentation of LIDAR point cloud data at difference height levels for 3D building extraction. IEEE International conference on Multimedia and Expo workshops (ICMEW) (Chengdu, 2014).

[8] Siddiqui, F. U., Mat-Isa, N. A., Yahya, A. 2013. Outlier rejection fuzzy c-means (ORFCM) algorithm for image segmentation. Turk. J. Electr. Eng. Co. 21, 6(2013) 18011819.

[9] Sohn, G., and Dowman, I. 2007. Data fusion of highresolution satellite imagery and LiDAR data for automatic building extraction. ISPRS J. Photogramm. 62(2007) 43-63. DOI= http://dx.doi.org/10.1016/j.isprsjprs.2007.01.001.

[10] Barista Software V.2.3 (2011).

[11] Lee, D. S., Shan, J., and Bethel, J. S. 2003. Class-guided building extraction from Ikonos imagery. Photogramm. Eng. Rem. S. 69, 2(Feb. 2003) 143-150. DOI=http://dx.doi.org/10.14358/PERS.69.2.143. 